PROCEEDINGS OF THE

AMERICAN MATHEMATICAL SOCIETY

Volume 139, Number 3, March 2011, Pages 1061-1072

S 0002-9939(2010)10647-0

Article electronically published on September 24, 2010

\title{
LINEARIZATION AND LOCAL STABILITY OF RANDOM DYNAMICAL SYSTEMS
}

\author{
IGOR V. EVSTIGNEEV, SERGEY A. PIROGOV, AND KLAUS R. SCHENK-HOPPÉ
}

(Communicated by Yingfei Yi)

\begin{abstract}
The paper examines questions of local asymptotic stability of random dynamical systems. Results concerning stochastic dynamics in general metric spaces, as well as in Banach spaces, are obtained. The results pertaining to Banach spaces are based on the linearization of the systems under study. The general theory is motivated (and illustrated in this paper) by applications in mathematical finance.
\end{abstract}

\section{Stochastic DYNAmics AND EQUILIBRIUM}

Let $(\Omega, \mathcal{F}, P)$ be a probability space and $T: \Omega \rightarrow \Omega$ its endomorphism, i.e., an $\mathcal{F}$-measurable map preserving the probability $P$ :

$$
P\left(T^{-1}(\Gamma)\right)=P(\Gamma), \Gamma \in \mathcal{F} .
$$

Let $(X, \mathcal{X})$ be a measurable space and $f(x, \omega)$ a jointly measurable mapping of $X \times \Omega$ into $X$. These data define a discrete-time (autonomous) random dynamical system with the state space $X$, the law of motion $f(x, \omega)$ and the time shift $T$. For each $t=1,2, \ldots$, we put

$$
f_{t}(x, \omega):=f\left(x, T^{t-1} \omega\right) .
$$

A sequence $x_{0}(\omega), x_{1}(\omega), \ldots$ of random elements in $X$ is called a path of the random dynamical system if

$$
x_{t}(\omega)=f_{t}\left(x_{t-1}(\omega), \omega\right), t=1,2, \ldots
$$

almost surely (a.s.). A random element $\bar{x}(\omega) \in X$ satisfying

$$
\bar{x}(T \omega)=f(\bar{x}(\omega), \omega)(\text { a.s. })
$$

is termed a stochastic equilibrium (or a random fixed point, or a stationary point) of the system. The sequence $\bar{x}_{t}(\omega):=\bar{x}\left(T^{t} \omega\right)$, which satisfies $\bar{x}_{t}(\omega)=f_{t}\left(\bar{x}_{t-1}(\omega), \omega\right)$ (a.s.) by virtue of (1.3), represents the equilibrium path of the system generated by the random fixed point $\bar{x}(\omega)$. For each $a \in X$, we denote by $x_{0}^{a}(\omega), x_{1}^{a}(\omega), \ldots$

Received by the editors March 29, 2010.

2010 Mathematics Subject Classification. Primary 37H05, 34F05; Secondary 91G80, 37H15.

Key words and phrases. Local stability, linearization, random fixed points, random dynamical systems, mathematical finance.

The authors gratefully acknowledge financial support from the Swiss National Center of Competence in Research "Financial Valuation and Risk Management" (project "Behavioural and Evolutionary Finance") and from the Finance Market Fund, Norway (projects "Stochastic Dynamics of Financial Markets" and "Stability of Financial Markets: An Evolutionary Approach").

(C)2010 American Mathematical Society Reverts to public domain 28 years from publication 
the path with the initial state $a$, i.e., the sequence of random elements generated recursively (for each $\omega$ and all $t \geq 1$ ) by equations (1.2) with $x_{0}(\omega)=a$.

\section{BASIC ASSUMPTIONS}

Let $\bar{x}(\omega)$ be a stochastic equilibrium and let $X(\omega) \subseteq X$ be a random set 1 such that $\bar{x}(\omega) \in X(\omega)$ and $f(x, \omega) \in X(T \omega)$ for each $x \in X(\omega)$ and $\omega \in \Omega$. Let $\rho$ be a metric on $X$. For any number $\delta>0$, define

$$
\bar{X}(\omega, \delta):=\{x \in X(\omega): \rho(x, \bar{x}(\omega)) \leq \delta\} .
$$

We introduce two hypotheses, $(\mathbf{A})$ and $(\mathbf{B})$, that will be needed for the statement of the main results.

(A) $X$ is a complete separable metric space with the metric $\rho(x, y)$ and the Borel $\sigma$-algebra $\mathcal{X}$. There exist random variables $L(\omega)>0$ and $\delta(\omega)>0$ such that

$$
\begin{gathered}
E|\ln L|<+\infty, E|\ln \delta|<+\infty, \\
E \ln L<0,
\end{gathered}
$$

and

$$
\rho(f(x, \omega), f(\bar{x}(\omega), \omega)) \leq L(\omega) \rho(x, \bar{x}(\omega))
$$

for all $x \in \bar{X}(\omega, \delta(\omega))$. Here, " $E$ " stands for the expectation with respect to the probability measure $P$.

It is assumed in $(\mathbf{A})$ that the mapping $f(\cdot, \omega)$ is locally Lipschitzian at the equilibrium point $\bar{x}(\omega)$ (in a $\delta(\omega)$-neighborhood of $\bar{x}(\omega)$ ) with the random Lipschitz constant $L(\omega)$. Conditions (2.1) guarantee that the random Lipschitz constant is not "too large" and the random $\delta(\omega)$-neighborhood is not "too small". (We could assume that $E \ln ^{+} L<+\infty$ and $E \ln ^{-} \delta>-\infty$, but this would not lead to a gain in generality.) According to (2.2), $L(\omega)$ has negative expected logarithm, so that the mapping $f(\cdot, \omega)$ is locally contracting on average.

The next hypothesis pertains to the case of a linear space $X$. It provides conditions guaranteeing the validity of $(\mathbf{A})$ formulated in terms of the linearization of the mapping $f(\cdot, \omega)$ at the equilibrium point $\bar{x}(\omega)$.

(B) $X$ is a separable Banach space with the norm $\|\cdot\|$, the metric $\rho(x, y):=$ $\|x-y\|$ and the Borel $\sigma$-algebra $\mathcal{X}$.

(B1) There exist random variables $L(\omega)>0$ and $\delta(\omega)>0$ for which conditions (2.1) hold and the mapping $f(x, \omega)$ satisfies (2.3) for all $x \in \bar{X}(\omega, \delta(\omega))$.

(B2) For each $\omega$, there exists the derivative $f^{\prime}(\bar{x}(\omega), \omega)$ of the mapping $f(\cdot, \omega)$ at the point $\bar{x}(\omega)$, and

$$
E \ln \left\|f^{\prime}(\bar{x}(\omega), \omega)\right\|<0 .
$$

The derivative is understood in the strong (Fréchet) sense, as a continuous linear operator $F(\omega):=f^{\prime}(\bar{x}(\omega), \omega)$ such that

$$
f(\bar{x}(\omega)+h, \omega)=f(\bar{x}(\omega), \omega)+F(\omega) h+g(h, \omega)\|h\|, h \in X,
$$

where $g(h, \omega) \rightarrow 0$ as $\|h\| \rightarrow 0$. The norm $\|F\|$ of the operator $F$ is defined as $\sup \{\|F h\| /\|h\|: 0 \neq h \in X\}$. Note that hypotheses (A) and (B1), in contrast with (B2), impose assumptions on the behavior of the mapping $f(x, \omega)$ only in the intersection of a neighborhood of the point $\bar{x}(\omega)$ with the set $X(\omega)$ (which might be significantly smaller than the whole neighborhood).

\footnotetext{
${ }^{1}$ We say that $X(\omega)$ is a random set if the graph $\{(u, \omega) \in X \times \Omega: u \in X(\omega)\}$ of the multivalued mapping $\omega \mapsto X(\omega)$ belongs to the $\sigma$-algebra $\mathcal{X} \otimes \mathcal{F}$.
} 
Throughout the paper we will assume that the endomorphism $T$ is ergodic: all invariant sets have either zero or full measure.

\section{Central result}

Theorem 1. Let hypothesis $(\boldsymbol{A})$ or hypothesis $(\boldsymbol{B})$ hold. Then there exist a random variable $\gamma(\omega)>0$ and a constant $c<0$ such that with probability one,

$$
\limsup _{t \rightarrow \infty} \frac{1}{t} \ln \rho\left(x_{t}^{a}(\omega), \bar{x}_{t}(\omega)\right) \leq c
$$

for all $a \in \bar{X}(\omega, \gamma(\omega))$.

The theorem shows that for all $\omega$ in a set $\Omega_{1}$ of measure 1 , the distance between the path $x_{t}^{a}(\omega)$ with the initial state $a$ and the equilibrium path $\bar{x}_{t}(\omega)$ tends to zero at an exponential rate for all $a$ in a sufficiently small neighborhood $\bar{X}(\omega, \gamma(\omega))$ of $\bar{x}(\omega)$. This convergence and its rate are uniform with respect to $\omega \in \Omega_{1}$ and $a \in \bar{X}(\omega, \gamma(\omega))$.

The results of this paper are motivated by applications in evolutionary finance 12. a new and rapidly developing area in financial mathematics. The general mathematical framework for models in this area is the ergodic theory of random dynamical systems. For the analysis of evolutionary models of asset markets, one often needs to examine local stability of stochastic equilibria of some dynamical systems. Surprisingly, the literature does not contain results which would be quite suitable for applications in this field. The main tools offered for local analysis in the context of stochastic dynamics (see the classical monograph by L. Arnold [1) are stochastic analogues of the Hartman-Grobman theorem [17 - 19] developed in [38] and [5] - 7], and closely related results on stable manifold theorems [4, 27, 29, 30, 34, 35. For the most part, these are delicate results, the use of which requires the verification of complex conditions. They are much stronger than what is needed for our purposes and require much stronger assumptions. The need for suitable tools motivated us to develop the techniques described above. To use Theorem 1 under hypothesis $(\mathbf{B})$ one has essentially to estimate only the expectation of one random variable: the logarithm of the norm of the derivative $f^{\prime}(x, \omega)$ at the random fixed point $x=\bar{x}(\omega)$ (the local Lipschitz property (B1) usually causes no problems). The theorem represents a direct stochastic analogue of well-known deterministic results on local asymptotic stability. Its statement and proof are based on elementary notions and techniques.

By and large, stochastic equilibria, or random fixed points, exist under sufficiently strong assumptions 2 In many cases such assumptions guarantee not only existence, but also global asymptotic stability established simultaneously as a consequence of a stochastic contraction principle [13, 36. Global asymptotic stability (convergence to a limit from any initial state) implies that the limit forms an equilibrium. Of course in such cases, the local analysis is superfluous. This is the situation, for example, with stochastic equilibrium problems related to random PerronFrobenius theorems $3,10,14,22,24$. Results in that field have been primarily inspired by the applications in the theory of Gibbs measures [33, 37, large deviations 23, models in mathematical biology 9 , and others (see also [20, 21, 25]). In many models coming from economics and finance, the situation is different. The questions of existence are often separated from the questions of stability, and existence

\footnotetext{
${ }^{2}$ Counterexamples in 14, 31 substantiate this assertion.
} 
proofs based on the above methods require too restrictive, unjustifiable assumptions. Typically, either the system has an obvious, even deterministic, fixed point, or the existence is proved by methods quite distinct from the above-mentioned arguments based on global stability and limiting procedures (see, e.g., studies on von Neumann-Gale dynamics [2, 15]). In the example we consider at the end of the paper, we deal with the former case: the system is random but the fixed point is deterministic.

The remainder of the paper is organized as follows. In section 4 we prove Theorem 1. At the end of the section, we provide a condition sufficient for the validity of hypothesis (B1). Section 5 gives an extension of Theorem 1 applicable both to continuous and discrete time settings. Sections 6 and 7 analyze an example related to mathematical finance.

\section{Proof of Theorem 1}

1st step. Assume that hypothesis (A) holds. Replacing $\Omega$ by its subset of full measure, we can (in view of (1.3) ) assume without loss of generality that the equations $\bar{x}_{t+1}(\omega)=f_{t}\left(\bar{x}_{t}(\omega), \omega\right)$ hold for all $t$ and all $\omega$, rather than almost surely. Define $X_{t}(\omega):=X\left(T^{t} \omega\right), \delta_{t}(\omega):=\delta\left(T^{t} \omega\right), L_{t}(\omega):=L\left(T^{t-1} \omega\right)$ and

$$
\bar{X}_{t}(\omega):=\left\{x \in X_{t}(\omega): \rho\left(x, \bar{x}_{t}(\omega)\right) \leq \delta_{t}(\omega)\right\} .
$$

It follows from (2.3) that

$$
\sup _{x \in \bar{X}_{t-1}(\omega)} \rho\left(f_{t}(x, \omega), \bar{x}_{t}(\omega)\right) \leq L_{t}(\omega) \rho\left(x, \bar{x}_{t-1}(\omega)\right) .
$$

This implies the validity of the following assertion:

$(*)$ If $x_{t-1}^{a}(\omega) \in \bar{X}_{t-1}(\omega)$, then

$$
\rho\left(x_{t}^{a}(\omega), \bar{x}_{t}(\omega)\right) \leq L_{t}(\omega) \rho\left(x_{t-1}^{a}(\omega), \bar{x}_{t-1}(\omega)\right) .
$$

We claim that there exists a random variable $\gamma(\omega)>0$ for which the inequalities

$$
\begin{gathered}
\gamma(\omega) \leq \delta_{0}(\omega), \\
L_{t}(\omega) \ldots L_{1}(\omega) \gamma(\omega) \leq \delta_{t}(\omega), t=1,2, \ldots
\end{gathered}
$$

hold with probability one. Indeed, these inequalities are satisfied if and only if

$$
\sigma(\omega):=\sup _{t=0,1, \ldots} \frac{L_{t}(\omega) \ldots L_{0}(\omega)}{\delta_{t}(\omega)} \leq \frac{1}{\gamma(\omega)}
$$

where $L_{0}(\omega):=1$. It is sufficient to show that $P\{\sigma<\infty\}=1$. Then relation (4.3), and hence inequalities (4.1) and (4.2), hold a.s. for the random variable $\gamma$ defined as $\gamma(\omega)=1 / \sigma(\omega)$ if $\sigma(\omega)<\infty$ and $\gamma(\omega)=1$ otherwise. To prove that $\sigma<\infty$ a.s., we use the ergodic theorem and obtain that

$$
\frac{1}{t} \ln \frac{L_{t} \ldots L_{0}}{\delta_{t}}=\frac{1}{t} \sum_{i=0}^{t} \ln L_{i}-\frac{1}{t} \ln \delta_{t} \rightarrow E \ln L<0 \text { (a.s.), }
$$

since $E|\ln L|<\infty$ and $E|\ln \delta|<\infty$. Consequently, $L_{t} \ldots L_{0} \delta_{t}^{-1} \rightarrow 0$ a.s., which implies that $P\{\sigma<\infty\}=1$. 
Consider the random variable $\gamma(\omega)>0$ constructed above and denote by $\Omega_{1}$ the set of those $\omega$ for which inequalities (4.1) and (4.2) hold and, additionally,

$$
\lim _{t \rightarrow \infty} \frac{1}{t} \sum_{i=0}^{t} \ln L_{i}=E \ln L .
$$

This relation holds a.s. by virtue of the ergodic theorem, and so $P\left(\Omega_{1}\right)=1$. Take any $\omega \in \Omega_{1}$ and $a \in X(\omega)$ satisfying $\rho(a, \bar{x}(\omega)) \leq \gamma(\omega)$. Let us show by induction that for all $t \geq 0$,

$$
\begin{gathered}
x_{t}^{a}(\omega) \in \bar{X}_{t}(\omega), \\
\rho\left(x_{t}^{a}(\omega), \bar{x}_{t}(\omega)\right) \leq L_{t}(\omega) \ldots L_{0}(\omega) \gamma(\omega) .
\end{gathered}
$$

For $t=0$, we have $a \in X_{0}(\omega)=X(\omega)$ by assumption and

$$
\rho(a, \bar{x}(\omega)) \leq \gamma(\omega) \leq \delta_{0}(\omega)
$$

by virtue of (4.1), so that $x_{0}^{a}(\omega) \in \bar{X}_{0}(\omega)$. Inequality (4.6) is true for $t=0$ in view of (4.7) and because $\gamma(\omega)=L_{0}(\omega) \gamma(\omega)$. Suppose relations (4.5) and (4.6) are valid for some $t$. Then $x_{t+1}^{a}(\omega) \in X_{t+1}(\omega)$ because

$$
\rho\left(x_{t+1}^{a}(\omega), \bar{x}_{t+1}(\omega)\right) \leq L_{t+1}(\omega) \rho\left(x_{t}^{a}(\omega), \bar{x}_{t}(\omega)\right) \leq L_{t+1}(\omega) L_{t}(\omega) \ldots L_{0}(\omega) \gamma(\omega),
$$

where the first inequality follows from assertion $(*)$ and (4.5), while the second is a consequence of (4.6). By using (4.2) (with $t+1$ in place of $t$ ), we conclude that $\rho\left(x_{t+1}^{a}(\omega), \bar{x}_{t+1}(\omega)\right) \leq \delta_{t+1}(\omega)$, which proves that the analogues of relations (4.5) and (4.6) hold for $t+1$. Inequality (4.6) combined with (4.4) implies (3.1) with $c:=E \ln L$.

2nd step. To complete the proof of Theorem 1 we show that $(\mathbf{B})$ implies $(\mathbf{A})$. In this connection, we make some comments regarding measurability. By applying (2.4) with $k^{-1} h$ in place of $h$, letting $k \rightarrow \infty$, and using the joint measurability of $f(x, \omega)$, we obtain that the mappings $F(\omega) h$ and $g(h, \omega)$, and hence the functions $\|F(\omega) h\|$ and $\|g(h, \omega)\|$, are jointly measurable in $(h, \omega)$. The operator norm $F(\omega)$ depends measurably on $\omega$ because $\|F(\omega)\|:=\sup _{k}\left\{\left\|F(\omega) h_{k}\right\| /\left\|h_{k}\right\|\right\}$, where $\left\{h_{k}\right\}$ is a countable dense subset in $X \backslash\{0\}$.

By using (2.4) and (2.3), we obtain that for each $x \in \bar{X}(\omega, \delta(\omega))$,

$$
\begin{gathered}
\|g(x-\bar{x}(\omega), \omega)\|\|x-\bar{x}(\omega)\| \leq\|f(x, \omega)-f(\bar{x}(\omega), \omega)\|+\|F(\omega)(x-\bar{x}(\omega))\| \\
\leq L(\omega)\|x-\bar{x}(\omega)\|+\|F(\omega)\|\|x-\bar{x}(\omega)\|,
\end{gathered}
$$

which implies

$$
\|g(x-\bar{x}(\omega), \omega)\| \leq L(\omega)+\|F(\omega)\| .
$$

Further, if $x \in \bar{X}(\omega, \delta(\omega))$, then

$$
\|f(x, \omega)-f(\bar{x}(\omega), \omega)\| \leq[\|F(\omega)\|+\|g(x-\bar{x}(\omega), \omega)\|]\|x-\bar{x}(\omega)\| .
$$

Define

$$
g_{k}(\omega):=\sup \left\{\|g(x-\bar{x}(\omega), \omega)\|: x \in \bar{X}\left(\omega, k^{-1} \delta(\omega)\right)\right\} .
$$

The function $g_{k}(\omega)$ is measurable with respect to the completion $\mathcal{F}^{P}$ of the $\sigma$ algebra $\mathcal{F}$ by $P$-null sets because $\|g(x-\bar{x}(\omega), \omega)\|$ is jointly measurable in $(x, \omega)$ and $X(\omega)$ is a random set. This follows from the fact that the projection of a set in $\mathcal{X} \otimes \mathcal{F}$ on $\Omega$ is $\mathcal{F}^{P}$-measurable (see, e.g., 8 , Theorem III.33). 
Define $D_{k}(\omega):=\|F(\omega)\|+g_{k}(\omega)$. By virtue of (2.4), $g_{k}(\omega) \rightarrow 0$ for each $\omega$. Furthermore, in view of (4.8) we have

$$
\ln D_{k}(\omega) \leq \ln [2\|F(\omega)\|+L(\omega)] \leq \ln 4+\max [\ln \|F(\omega)\|, \ln L(\omega)]=: \Xi(\omega),
$$

where $E|\Xi(\omega)|<\infty$. By using Fatou's lemma, we get

$$
\lim \sup _{k \rightarrow \infty} E \ln D_{k}(\omega) \leq E \lim _{k \rightarrow \infty} \ln D_{k}(\omega)=E \ln \|F(\omega)\|<0 .
$$

Thus there exists $k$ such that $E \ln D_{k}(\omega)<0$ and (by virtue of (4.9) and (4.10))

$$
\|f(x, \omega)-f(\bar{x}(\omega), \omega)\| \leq D_{k}(\omega)\|x-\bar{x}(\omega)\|
$$

for each $x \in \bar{X}(\omega, \delta(\omega) / k)$. This completes the proof of Theorem 1

Remark 1. The following assumption is sufficient for condition (B1) to hold.

(B3) There exist random variables $L(\omega)>0$ and $\delta(\omega)>0$ satisfying (2.1) such that for all $x \in \bar{X}(\omega, \delta(\omega))$, the set $X(\omega)$ contains the segment $[\bar{x}(\omega), x]$ connecting $\bar{x}(\omega)$ and $x$, the mapping $f(x, \omega)$ is differentiable at the point $x$, and the norm of the derivative $\left\|f^{\prime}(x, \omega)\right\|$ is bounded from above by $L(\omega)$.

To deduce (B1) from (B3) it suffices to observe that the inequality $\left\|f^{\prime}(x, \omega)\right\| \leq$ $L(\omega)$ implies (2.3) by virtue of the generalized mean value theorem:

$$
\|f(x, \omega)-f(\bar{x}(\omega), \omega)\| \leq\|x-\bar{x}(\omega)\| \sup _{y \in[\bar{x}(\omega), x]}\left\|f^{\prime}(y, \omega)\right\|
$$

(which holds even if $f^{\prime}(x, \omega)$ is the weak rather than the strong derivative); see [26], Section X.1.3.

\section{Extension to Discrete AND CONTINuOUS TIME COCYCLES}

The next result provides an extension of Theorem 1 to random dynamical systems defined in terms of cocycles in discrete and continuous time. Let $\mathbb{T}$ be either the set of non-negative integers or the set of non-negative real numbers, and let $T^{t}, t \in \mathbb{T}$, be a semigroup of ergodic endomorphisms of the probability space $(\Omega, \mathcal{F}, P)$. For each $t \in \mathbb{T}$, let $C_{t}(x, \omega)$ be a jointly measurable mapping of $X \times \Omega$ into $X$. Assume that the family of mappings $C_{t}(x, \omega), t \in \mathbb{T}$, forms a cocycle, i.e.,

$$
C_{t+s}(\cdot, \omega)=C_{s}\left(\cdot, T^{t} \omega\right) \circ C_{t}(\cdot, \omega), C_{0}(x, \omega)=x,
$$

for all $t, s, x$ and $\omega$. The cocycle defines the law of motion in the system, whose paths are random functions $x_{t}(\omega), t \in \mathbb{T}$, such that with probability one, $x_{t}(\omega)=$ $C_{t}\left(x_{0}(\omega), \omega\right)$ for all $t \in \mathbb{T}$. Random dynamical systems of this kind can be generated by stochastic or random differential equations in continuous time and by products of random mappings in discrete time (see [1]). We will assume that a random set $X(\omega)$ is given such that $C_{t}(x, \omega) \in X\left(T^{t} \omega\right)$ for all $x \in X(\omega)$.

Let $\bar{x}_{t}(\omega), t \in \mathbb{T}$, be an equilibrium path, i.e., a path satisfying a.s. $\bar{x}_{t}(\omega)=$ $\bar{x}_{0}\left(T^{t} \omega\right)$ for all $t \in \mathbb{T}$. For each $a \in X$, define $x_{t}^{a}(\omega):=C_{t}(a, \omega)$ (the random path with the initial state $a$ ).

Theorem 2. Let the following assumptions hold:

(C1) There exists $M \in \mathbb{T}$ such that the mapping $C_{M}(\cdot, \omega)$ satisfies condition $(\mathbf{A})$ or (B) with $\bar{x}(\omega)=\bar{x}_{0}(\omega)$.

(C2) There are random variables $H(\omega)>0, \kappa(\omega)>0$ and a constant $b>0$ such that $E|\ln H(\omega)|<+\infty, E|\ln \kappa(\omega)|<+\infty$ and with probability one,

$$
\rho\left(C_{t}(x, \omega), C_{t}(\bar{x}(\omega), \omega)\right) \leq H(\omega) \rho(x, \bar{x}(\omega))^{b}
$$


for all $x \in \bar{X}(\omega, \kappa(\omega))$ and $t \in \mathbb{T}$ satisfying $0 \leq t \leq M$.

Then there exist a random variable $\gamma(\omega)>0$ and a constant $d<0$ such that almost surely

$$
\limsup _{\mathbb{T} \ni t \rightarrow \infty} \frac{1}{t} \ln \rho\left(x_{t}^{a}(\omega), \bar{x}_{t}(\omega)\right) \leq d
$$

for all $a \in \bar{X}(\omega, \gamma(\omega))$.

Since $d<0$, (5.2) implies that $\rho\left(x_{t}^{a}(\omega), \bar{x}_{t}(\omega)\right) \rightarrow 0$ a.s. at an exponential rate. This convergence, as well as its rate, are uniform with respect to $\omega$ in a set $\Omega_{1}$ of full measure and all $a$ in the neighborhood $\bar{X}(\omega, \gamma(\omega))$ of $\bar{x}(\omega)=\bar{x}_{0}(\omega)$. Property (5.1) represents a Hölder condition on the cocycle $C_{t}(x, \omega)$.

Proof of Theorem 2. Since (B) implies (A), as we demonstrated in section 4. it is sufficient to prove the theorem under assumption $(\mathbf{A})$. Define $t(n):=n M$, $n=0,1, \ldots$ By applying Theorem 1 to the mapping $f(x, \omega):=C_{M}(x, \omega)$, we obtain that there exist a random variable $\gamma(\omega)>0$ and a constant $c<0$ such that with probability one,

$$
\limsup _{n \rightarrow \infty} \frac{1}{t(n)} \ln \rho\left(x_{t(n)}^{a}(\omega), \bar{x}_{t(n)}(\omega)\right) \leq c
$$

for all $a \in \bar{X}(\omega, \gamma(\omega))$. From (5.3) we obtain that for each $\varepsilon \in(0,1)$ with probability one,

$$
\rho_{n}(\omega):=\rho\left(x_{t(n)}^{a}(\omega), \bar{x}_{t(n)}(\omega)\right) \leq \exp [t(n) c(1-\varepsilon)]
$$

for all sufficiently large $n$. Furthermore, almost surely

$$
\kappa_{n}(\omega):=\kappa\left(T^{t(n)} \omega\right) \geq \exp [t(n) c(1-\varepsilon)]
$$

for all $n$ large enough. Indeed, the last inequality holds if $M c(1-\varepsilon) \leq n^{-1} \ln \kappa_{n}(\omega)$, which is true for all $n$ large enough because $\lim _{n \rightarrow \infty} n^{-1} \ln \kappa_{n}(\omega) \rightarrow 0$ a.s. (this follows from the assumption $E|\ln \kappa(\omega)|<+\infty$ ). From (5.4) and (5.5) we obtain that the inequalities

$$
\rho_{n}(\omega) \leq \kappa_{n}(\omega)
$$

a.s. hold for all $n$ large enough. By using (C2) and (5.6), we obtain that for each $\varepsilon>0$ with probability one,

$$
\begin{gathered}
\eta_{n}(\omega):=\sup _{t(n) \leq t \leq t(n+1)} \frac{1}{t} \ln \rho\left(x_{t}^{a}(\omega), \bar{x}_{t}(\omega)\right) \\
\leq \sup _{t(n) \leq t \leq t(n+1)}\left[\frac{\ln ^{+} H\left(T^{t(n)} \omega\right)}{t}+\frac{b}{t} \ln \rho\left(x_{t(n)}^{a}(\omega), \bar{x}_{t(n)}(\omega)\right)\right] \\
\leq \frac{\ln ^{+} H\left(T^{t(n)} \omega\right)}{t(n)}+\sup _{t(n) \leq t \leq t(n+1)} \frac{b t(n) c(1-\varepsilon)}{t}=\frac{\ln ^{+} H\left(T^{t(n)} \omega\right)}{t(n)}+\frac{b n c(1-\varepsilon)}{n+1}
\end{gathered}
$$

for all $n$ greater than some $n(\omega)$. The first summand in the last expression converges to zero a.s. by virtue of the assumption $E|\ln H|<+\infty$. The second summand tends to $b c(1-\varepsilon)$. Consequently, with probability one, there exists $k(\omega)$ such that $\eta_{n}(\omega) \leq b c(1-\varepsilon)^{2}$ for all $n \geq k(\omega)$. Denote by $N(t)$ the natural number such that $M N(t) \leq t<M[N(t)+1]$. Then $t^{-1} \ln \rho\left(x_{t}^{a}(\omega), \bar{x}_{t}(\omega)\right) \leq \eta_{N(t)}(\omega)$. Thus if $t \geq M k(\omega)$, then $N(t) \geq k(\omega)$, and so

$$
t^{-1} \ln \rho\left(x_{t}^{a}(\omega), \bar{x}_{t}(\omega)\right) \leq \eta_{N(t)}(\omega) \leq b c(1-\varepsilon)^{2} .
$$


Since $\varepsilon$ is any number in $(0,1)$, we obtain that (5.2) holds with $d:=b c(<0)$. The proof is complete.

Remark 2. Applying Theorem 2 in the discrete-time case, where $\mathbb{T}=\{0,1, \ldots\}$, to the cocycle defined by

$$
C_{t}(\cdot, \omega):=f_{t}(\cdot, \omega) \circ \ldots \circ f_{1}(\cdot, \omega), t \geq 1
$$

we obtain a version of Theorem 1 in which condition $(\mathbf{A})$ or $(\mathbf{B})$ is imposed not on the given mapping $f(\cdot, \omega)$, but on the product $C_{M}(\cdot, \omega)=f_{M}(\cdot, \omega) \circ \ldots \circ f_{1}(\cdot, \omega)$ of the mappings $f_{t}(\cdot, \omega)$. In this case, hypothesis $(\mathbf{C 2})$ is fulfilled under the following assumption.

(C3) There exist random variables $L(\omega)>0$ and $\delta(\omega)>0$ for which conditions (2.1) and (2.3) hold.

Indeed, assume that $(\mathbf{C 3})$ is satisfied and put

$$
\kappa(\omega):=\min _{0 \leq t \leq M} \frac{\delta_{t}(\omega)}{L_{0}(\omega) \ldots L_{t}(\omega)},
$$

where $L_{t}$ and $\delta_{t}$ are defined in section 4. Arguing by induction and using (2.3), we obtain that

$$
\rho\left(C_{t}(x, \omega), C_{t}(\bar{x}(\omega), \omega)\right) \leq L_{0}(\omega) \ldots L_{t}(\omega) \rho(x, \bar{x}(\omega)) \leq \delta_{t}(\omega)
$$

$(t=1,2, \ldots, M)$, as long as $\rho(x, \bar{x}(\omega)) \leq \kappa(\omega)$. This yields $(\mathbf{C 2})$ with $b=1$ and $H(\omega):=L_{1}(\omega) \ldots L_{M}(\omega)$.

Remark 3. Assume that $X$ is a separable Banach space and for each $\omega$, the mapping $C_{t}(\cdot, \omega)$ is differentiable at the point $\bar{x}_{0}(\omega)$ and continuous in a neighborhood of this point. Then the family of linear operators $F_{t}(\omega)=C_{t}^{\prime}\left(\bar{x}_{0}(\omega), \omega\right)$ forms a cocycle (this follows from the chain rule of differentiation), called the linearization of the cocycle $C_{t}(x, \omega)$. Suppose that $E \ln ^{+}\left\|F_{t}(\omega)\right\|<+\infty$. In this case, the assumption that $E \ln \left\|F_{M}(\omega)\right\|<0$ for some $M>0$, needed for the application of Theorem 2 under hypothesis (B), is equivalent to the assumption that the Furstenberg-Kesten constant [16, 32, 1]

$$
\lim _{t \rightarrow \infty} \frac{1}{t} E \ln \left\|F_{t}(\omega)\right\|=\inf _{t>0} \frac{1}{t} E \ln \left\|F_{t}(\omega)\right\|
$$

is negative.

\section{Application to an investment model}

To describe an example to which we will apply Theorem 1, assume that together with the probability space $(\Omega, \mathcal{F}, P)$ and its endomorphism $T$, we are given a family of $\sigma$-algebras $\mathcal{F}_{0} \subseteq \mathcal{F}_{1} \subseteq \ldots \subseteq \mathcal{F}$ such that $T^{-1}(\Gamma) \in \mathcal{F}_{t+1}$ if and only if $\Gamma \in \mathcal{F}_{t}$ $\left(\mathcal{F}_{t}\right.$ contains events observable prior to time $\left.t\right)$. Denote by $\Delta$ the unit simplex $\left\{v=\left(v_{1}, \ldots, v_{K}\right) \geq 0: \sum v_{k}=1\right\}$. Let $R_{t}(\omega)=R\left(T^{t} \omega\right), \lambda_{t}(\omega)=\lambda\left(T^{t} \omega\right)$ and $\lambda_{t}^{*}(\omega)=\lambda^{*}\left(T^{t} \omega\right)$ be stationary processes with values in $\Delta$ adapted to the filtration $\left(\mathcal{F}_{t}\right)$ and $r$ a number in $(0,1)$ such that

$$
r E_{t} \lambda^{*}(T \omega)+(1-r) E_{t} R(T \omega)=\lambda^{*}(\omega) \text { (a.s.), }
$$

where $E_{t}(\cdot)=E\left(\cdot \mid \mathcal{F}_{t}\right)$. The existence and uniqueness of the solution $\lambda^{*}(\cdot)$ to equation (6.1) follows from the Banach contraction principle and the fact that $r<1$. 
Consider the random dynamical system whose paths $\left(x_{t}\right)$ are defined by

$$
x_{t+1}=x_{t} \frac{\sum_{k=1}^{K}\left[r \lambda_{t+1, k}^{*}+(1-r) R_{t+1, k}\right] \frac{\lambda_{t, k}}{\lambda_{t, k} x_{t}+\lambda_{t, k}^{*}}}{\sum_{k=1}^{K}\left[r \lambda_{t+1, k}+(1-r) R_{t+1, k}\right] \frac{\lambda_{t, k}^{*}}{\lambda_{t, k} x_{t}+\lambda_{t, k}^{*}}},
$$

where $R_{t, k}, \lambda_{t, k}$, and $\lambda_{t, k}^{*}$ are the coordinates of the vectors $R_{t}, \lambda_{t}$, and $\lambda_{t}^{*}$, respectively. In the evolutionary model of an asset market developed in 11, 12] (see these references for details), $R_{t, k}$ are relative dividends of $K$ assets, the sequences of vectors $\lambda=\left(\lambda_{t}\right)$ and $\lambda^{*}=\left(\lambda_{t}^{*}\right)$ are investment strategies (portfolio rules) and $r$ is the investment rate. The vectors $\lambda_{t} \in \Delta$ and $\lambda_{t}^{*} \in \Delta$ indicate proportions according to which investors using the strategies $\lambda$ and $\lambda^{*}$ allocate wealth across assets. The strategy $\lambda^{*}$ defined by (6.1) is a generalization of the Kelly portfolio rule, well-known in mathematical finance (see, e.g., [28]). It is assumed that there are two groups of investors, one using the strategy $\lambda^{*}$ and the other any strategy $\lambda$ distinct from $\lambda^{*}$. The variable $x_{t}$ represents the ratio $w_{t} / w_{t}^{*}$ where $w_{t}^{*}$ and $w_{t}$ denote the total wealth of the former and the latter groups of investors, respectively. The local stability of the dynamical system under consideration at the fixed point $\bar{x}=0$ means that the portfolio rule $\lambda^{*}$ is evolutionary stable. If the initial relative wealth $x_{0}=a$ of the $\lambda$-investors ("mutants" in the terminology borrowed from biology) is small enough, then they will be eventually driven out of the market by the $\lambda^{*}$-investors: their relative wealth $x_{t}^{a}$ will tend to zero.

Define $\lambda_{k}^{*}:=\lambda_{0, k}^{*}$ and assume that the following conditions hold.

(K1) $E \ln \min _{k} \lambda_{k}^{*}>-\infty$.

(K2) The random variables $\mu_{k}:=r \lambda_{1, k}^{*}+(1-r) R_{1, k}, k=1, \ldots, K$, are conditionally linearly independent given $\mathcal{F}_{0}$, i.e., the equality $\alpha_{1} \mu_{1}+\ldots+\alpha_{K} \mu_{K}=0$ holding (a.s.) for some $\mathcal{F}_{0}$-measurable random variables $\alpha_{k}$ implies $\alpha_{1}=\ldots=\alpha_{K}=0$ (a.s.).

Theorem 3. For any strategy $\lambda \neq \lambda^{*}$, there exist a random variable $\gamma(\omega)>0$ and a constant $c<0$ such that with probability one, $\limsup _{t \rightarrow \infty} t^{-1} \ln x_{t}^{a} \leq c$ for all $0 \leq a \leq \gamma(\omega)$.

Proof. We apply Theorem 1 with $X=(-\infty,+\infty)$ and $X(\omega)=[0, \infty)$. We define the function $f(x, \omega)$ as the right-hand side of (6.2) with $t=0$ if $x=x_{t}>-\zeta(\omega)$, where $\zeta(\omega):=\min _{k} \lambda_{k}^{*}(\omega)$. For $x \leq-\zeta(\omega)$, we can define $f(x, \omega)$, for example, as any (fixed) number $u$. Clearly $\bar{x}:=0$ is a fixed point of $f(x, \omega)$ for each $\omega$. For $0<x \leq 1$, we have $0<x^{-1} f(x, \omega) \leq 2 \zeta^{-2}$, so that (B1) holds with $\delta=1$ and $L=2 \zeta^{-2}$. Further,

$$
f^{\prime}(0, \omega)=\sum_{k=1}^{K} \mu_{k} \frac{\lambda_{k}}{\lambda_{k}^{*}}
$$

By virtue of Jensen's inequality, we have

$$
E \ln \sum_{k=1}^{K} \mu_{k} \frac{\lambda_{k}}{\lambda_{k}^{*}}=E\left(E_{0} \ln \sum_{k=1}^{K} \mu_{k} \frac{\lambda_{k}}{\lambda_{k}^{*}}\right)<E\left(\ln \sum_{k=1}^{K} E_{0} \mu_{k} \frac{\lambda_{k}}{\lambda_{k}^{*}}\right)=E \ln \sum_{k=1}^{K} \lambda_{k}=0 .
$$

To show that the above inequality is indeed strict, assume the contrary. Then the random variable $\sum_{k=1}^{K} \mu_{k} \lambda_{k} / \lambda_{k}^{*}$ coincides a.s. with an $\mathcal{F}_{0}$-measurable random variable. Hence it coincides a.s. with its conditional expectation given $\mathcal{F}_{0}$, which is 
equal to 1 (this follows from (6.1)). Thus $\sum_{k=1}^{K} \mu_{k} \lambda_{k} / \lambda_{k}^{*}=1$ (a.s.) or, equivalently, $\sum_{k=1}^{K} \mu_{k}\left[\left(\lambda_{k} / \lambda_{k}^{*}\right)-1\right]=0$ (a.s.), which implies by virtue of $(\mathbf{K 2})$ that $\left(\lambda_{k} / \lambda_{k}^{*}\right)-1=$ 0 (a.s.) for all $k=1,2, \ldots, K$. Consequently, $\lambda=\lambda^{*}$, which is a contradiction.

\section{Sufficient conditions in the Markovian Case}

We conclude with some comments regarding hypotheses $(\mathbf{K 1})$ and (K2). These hypotheses are formulated in terms of the Kelly strategy $\lambda^{*}$, which is defined as the solution to equation (6.1). For the applications it is important to provide conditions sufficient for (K1) and (K2) that are formulated in terms of one of the primitives of the model, namely the dividend process $R_{t}$. The former condition holds if $E \ln \min _{k} E_{0} R_{1, k}>-\infty$, which is clear from (6.1). The latter is satisfied, for example, if the following requirements are fulfilled:

(i) the random variables $R_{1, k}, k=1, \ldots, K$, are conditionally independent given $\mathcal{F}_{0}$ (the absence of "redundant" assets);

(ii) the probability space $(\Omega, \mathcal{F}, P)$ and the filtration $\left(\mathcal{F}_{t}\right)$ are generated by a stationary Markov process ..., $s_{-1}, s_{0}, s_{1}, \ldots$ with values in a measurable space $S$, and the vector function $R(\omega)$ depends only on $s_{0}$ (we shall denote it as $R\left(s_{0}\right)$ );

(iii) the process $s_{t}$ has a transition function $\pi(s, d \sigma)$ possessing a jointly measurable density $p(s, \sigma)$ with respect to a probability measure $\pi(d \sigma)$ such that $0<v \leq p(s, \sigma) \leq V$ for some constants $v \leq V$.

Let us prove that conditions (i)-(iii) imply (K2). We first observe that in the Markov case, $\lambda_{k}^{*}=\lambda_{k}^{*}\left(s_{0}\right)$ and $\mu_{k}=\mu_{k}\left(s_{1}\right)$ are functions of $s_{0}$ and $s_{1}$, respectively. It follows from (6.1) that $\mu$ satisfies

$$
r E_{0} \mu\left(s_{1}\right)+(1-r) R\left(s_{0}\right)=\mu\left(s_{0}\right) \text { (a.s.). }
$$

In the present setting, $\mathcal{F}_{t}$-measurable functions can be identified with measurable functions $\alpha\left(s^{t}\right)$, where $s^{t}:=\left(\ldots, s_{t-1}, s_{t}\right)$. Let $\alpha_{1}\left(s^{0}\right), \ldots, \alpha_{K}\left(s^{0}\right)$ be $\mathcal{F}_{0}$-measurable vector functions satisfying

$$
\left\langle\alpha\left(s^{0}\right), \mu\left(s_{1}\right)\right\rangle=0 \text { (a.s.), }
$$

where $\alpha:=\left(\alpha_{1}, \ldots, \alpha_{K}\right)$ and $\mu=\left(\mu_{1}, \ldots, \mu_{K}\right)$. We have to prove that $\alpha=0$ (a.s.). From (7.1) we get

$$
r\left\langle\alpha\left(s^{-1}\right), E_{0} \mu\left(s_{1}\right)\right\rangle+(1-r)\left\langle\alpha\left(s^{-1}\right), R\left(s_{0}\right)\right\rangle=\left\langle\alpha\left(s^{-1}\right), \mu\left(s_{0}\right)\right\rangle \text { (a.s.). }
$$

Let us show that $E\left|\left\langle\alpha\left(s^{-1}\right), E_{0} \mu\left(s_{1}\right)\right\rangle\right|=0$. We have

$$
\begin{gathered}
E\left|\left\langle\alpha\left(s^{-1}\right), E_{0} \mu\left(s_{1}\right)\right\rangle\right|=E\left|E_{0}\left\langle\alpha\left(s^{-1}\right), \mu\left(s_{1}\right)\right\rangle\right| \leq E E_{0}\left|\left\langle\alpha\left(s^{-1}\right), \mu\left(s_{1}\right)\right\rangle\right| \\
=E\left|\left\langle\alpha\left(s^{-1}\right), \mu\left(s_{1}\right)\right\rangle\right|=E E_{-1}\left|\left\langle\alpha\left(s^{-1}\right), \mu\left(s_{1}\right)\right\rangle\right| .
\end{gathered}
$$

From (iii) we get $p(s, \sigma) \leq V v^{-1} p\left(s_{-1}, \sigma\right)$. By using this, we obtain

$$
\begin{gathered}
E_{-1}\left|\left\langle\alpha\left(s^{-1}\right), \mu\left(s_{1}\right)\right\rangle\right|=\int \pi\left(s_{-1}, d s\right) \int \pi(s, d \sigma)\left|\left\langle\alpha\left(s^{-1}\right), \mu(\sigma)\right\rangle\right| \\
=\int \pi\left(s_{-1}, d s\right) \int p(s, \sigma) \pi(d \sigma)\left|\left\langle\alpha\left(s^{-1}\right), \mu(\sigma)\right\rangle\right| \\
\leq V v^{-1} \int \pi\left(s_{-1}, d s\right) \int p\left(s_{-1}, \sigma\right) \pi(d \sigma)\left|\left\langle\alpha\left(s^{-1}\right), \mu(\sigma)\right\rangle\right| \\
=V v^{-1} \int \pi\left(s_{-1}, d \sigma\right)\left|\left\langle\alpha\left(s^{-1}\right), \mu(\sigma)\right\rangle\right|=V v^{-1} E_{-1}\left|\left\langle\alpha\left(s^{-1}\right), \mu\left(s_{0}\right)\right\rangle\right| .
\end{gathered}
$$


By combining (7.5), (7.4) and (7.2), we obtain that $E\left|\left\langle\alpha\left(s^{-1}\right), E_{0} \mu\left(s_{1}\right)\right\rangle\right|=0$. This, together with (17.2) and (7.3), implies the equality $\left\langle\alpha\left(s^{-1}\right), R\left(s_{0}\right)\right\rangle=0$ (a.s.). By using (i) we conclude that $\alpha\left(s^{-1}\right)=0$ (a.s.), or, equivalently, $\alpha\left(s^{0}\right)=0$ (a.s.), which completes the proof.

\section{REFERENCES}

[1] L. Arnold. Random Dynamical Systems. Springer, 1998. MR.1723992 (2000m:37087)

[2] L. Arnold, I. V. Evstigneev and V. M. Gundlach. Convex-valued random dynamical systems: A variational principle for equilibrium states. Random Oper. Stoch. Equ. 7 (1999), 23-38. MR:1677758 (2000j:60078)

[3] L. Arnold, V. M. Gundlach and L. Demetrius. Evolutionary formalism for products of positive random matrices. Ann. Appl. Probab. 4 (1994), 859-901. MR.1284989 (95h:28028)

[4] A. Carverhill. Flows of stochastic dynamical systems: Ergodic theory. Stochastics 14 (1985), 273-317. MR805125 (87c:58059)

[5] E. A. Coayla-Teran, S.-E. A. Mohammed and P. R. C. Ruffino. Hartman-Grobman theorems along hyperbolic stationary trajectories. Discrete Contin. Dyn. Syst. 17 (2007), 281-292. MR2257433 (2007j:37084)

[6] E. A. Coayla-Teran and P. R. C. Ruffino. Stochastic versions of Hartman-Grobman theorems. Stoch. Dyn. 4 (2004), 571-593. MR2102754 (2005g:37101)

[7] N. D. Cong. Topological classification of linear hyperbolic cocycles. J. Dynam. Differential Equations 8 (1996), 427-467. MR1412246 (97j:58117)

[8] C. Dellacherie and P.-A. Meyer. Probabilities and Potential. North-Holland, 1978. MR.521810 (80b:60004)

[9] L. Demetrius and V. M. Gundlach. Evolutionary dynamics in random environments. In: H. Crauel and V. M. Gundlach (eds.), Stochastic Dynamics. Springer, 1999, pp. 371-394. MR:1678507 (99m:58176)

[10] I. V. Evstigneev. Positive matrix-valued cocycles over dynamical systems. Uspekhi Matem. Nauk (Russ. Math. Surveys) 29, No. 5 (1974), 219-220. (In Russian) MR0396906 (53:766)

[11] I. V. Evstigneev, T. Hens and K. R. Schenk-Hoppé. Evolutionary stable stock markets. Econom. Theory 27 (2006), 449-468. MR2212761 (2007h:91087)

[12] I. V. Evstigneev, T. Hens and K. R. Schenk-Hoppé. Evolutionary finance. In: T. Hens and K. R. Schenk-Hoppé (eds.), Handbook of Financial Markets: Dynamics and Evolution. NorthHolland, 2009, pp. 507-566.

[13] I. V. Evstigneev and S. A. Pirogov. A stochastic contraction principle. Random Oper. Stoch. Equ. 15 (2007), 155-162. MR2337912(2008h:47115)

[14] I. V. Evstigneev and S. A. Pirogov. Stochastic nonlinear Perron-Frobenius theorem. Positivity 14 (2010), 43-57. MR2596462

[15] I. V. Evstigneev and K. R. Schenk-Hoppé. Stochastic equilibria in von Neumann-Gale dynamical systems. Trans. Amer. Math. Soc. 360 (2008), 3345-3364. MR2379800 (2010b:91135)

[16] H. Furstenberg and H. Kesten. Products of random matrices. Ann. Math. Statist. 31 (1960), 457-469. MR0121828 (22:12558)

[17] D. M. Grobman. Homeomorphisms of systems of differential equations. Dokl. Akad. Nauk SSSR 128 (1959), 880-881. MR0121545 (22:12282)

[18] P. Hartman. A lemma in the theory of structural stability of differential equations. Proc. Amer. Math. Soc. 11 (1960), 610-620. MR0121542 (22:12279)

[19] P. Hartman. On local homeomorphisms of Euclidean spaces. Bol. Soc. Mat. Mexicana 5 (1960), 220-241. MR0141856 (25:5253)

[20] K. Khanin and Yu. Kifer. Thermodynamic formalism for random transformations and statistical mechanics. Amer. Math. Soc. Transl. Ser. 2171 (1996), 107-140. MR1359097|(96j:58136)

[21] Yu. Kifer. Fractal dimensions and random transformations. Trans. Amer. Math. Soc. 348 (1996), 2003-2038. MR1348865 (96i:28009)

[22] Yu. Kifer. Perron-Frobenius theorem, large deviations, and random perturbations in random environments. Math. Z. 222 (1996), 677-698. MR1406273 (97f:60131)

[23] Yu. Kifer. Limit theorems for random transformations and processes in random environments. Trans. Amer. Math. Soc. 350 (1998), 1481-1518. MR1451607(98i:60021)

[24] Yu. Kifer. Thermodynamic formalism for random transformations revisited. Stoch. Dyn. 8 (2008), 77-102. MR2399927(2009a:37066) 
[25] Yu. Kifer and P.-D. Liu. Random dynamics. In: B. Hasselblatt and A. Katok (eds.), Handbook of Dynamical Systems, Vol. 1B. Elsevier, 2006, pp. 379-499. MR2186245 (2008a:37002)

[26] A. N. Kolmogorov and S. V. Fomin. Elements of the Theory of Functions and Functional Analysis. Graylock, 1957. MR.0085462 (19:44d)

[27] Z. Lian and K. Lu. Lyapunov exponents and invariant manifolds for random dynamical systems in a Banach space. Mem. Amer. Math. Soc. 206 (2010), no. 967.

[28] L. C. MacLean, E. O. Thorp and W. T. Ziemba (eds.). The Kelly Capital Growth Investment Criterion: Theory and Practice. World Scientific, 2011.

[29] S.-E. A. Mohammed and M. K. R. Scheutzow. The stable manifold theorem for stochastic differential equations. Ann. Probab. 27 (1999), 615-652. MR.1698943(2001e:60121)

[30] S.-E. A. Mohammed, T. Zhang and H. Zhao. The stable manifold theorem for semilinear stochastic evolution equations and stochastic partial differential equations. Mem. Amer. Math. Soc. 196 (2008), no. 917. MR.2459571 (2010b:60175)

[31] G. Ochs and V. I. Oseledets. Topological fixed point theorems do not hold for random dynamical systems. J. Dynam. Differential Equations 11 (1999), 583-593. MR1725412 (2000i:37075)

[32] V. I. Oseledec. A multiplicative ergodic theorem: Characteristic Ljapunov exponents of dynamical systems. Trans. Moscow Math. Soc. 19 (1968), 197-231. MR0240280 (39:1629)

[33] S. A. Pirogov and Ya. G. Sinai. Phase diagrams of classical lattice systems, I and II, Theor. Math. Phys. 25 (1975), 1185-1192; III, Theor. Math. Phys. 26 (1976), 39-49. MR0676316 $(58: 32680)$ MR0676499 (58:32712)

[34] D. Ruelle. Ergodic theory of differentiable dynamical systems. Publ. Math. Inst. Hautes Études Sci. 50 (1979), 27-58. MR556581 (81f:58031)

[35] D. Ruelle. Characteristic exponents and invariant manifolds in Hilbert space. Ann. of Math. (2) 115 (1982), 243-290. MR647807 (83j:58097)

[36] B. Schmalfuss. A random fixed point theorem and the random graph transformation. J. Math. Anal. Appl. 225 (1998), 91-113. MR1639297(99i:47118)

[37] Ya. G. Sinai. Gibbs measures in ergodic theory. Uspekhi Matem. Nauk (Russ. Math. Surveys) 27 (1972), 21-69. MR0399421 (53:3265)

[38] T. Wanner. Linearization of random dynamical systems. In: C. John, U. Kirchgraber and H. O. Walther (eds.), Dynamics Reported, Vol. 4. Springer, 1994, pp. 203-269. MR 1346499 (96m:34084)

Department of Economics, University of Manchester, Manchester M13 9PL, United KINGDOM

E-mail address: igor.evstigneev@manchester.ac.uk

Institute for Information Transmission Problems, Academy of Sciences of Russia, GSP-4, Moscow, 101447, Russia

E-mail address: pirogov@mail.ru

School of Mathematics and Leeds University Business School, University of Leeds, LEEDS LS2 9JT, United Kingdom

E-mail address: k.r.schenk-hoppe@leeds.ac.uk 